\title{
Concreteness effects in free recall: The roles of imaginal and relational processing
}

\author{
MARC MARSCHARK \\ University of North Carolina at Greensboro, Greensboro, North Carolina \\ and \\ LUCA SURIAN \\ MRC Cognitive Development Unit, London, England
}

\begin{abstract}
Two experiments reevaluated the possible role of mental imagery in free recall of concrete and abstract words. In Experiment 1, the number and rate of list presentations were manipulated. Incidental recall following an imagery rating task yielded reliable concreteness effects after two presentations but not after a single presentation, regardless of presentation rate. In Experiment 2, we examined the effects of relational (categorization) and item-specific (imagery rating) processing tasks on memory for categorically related or unrelated concrete and abstract words. Concreteness effects were obtained when unrelated words were sorted into categories but not when they were rated on imagery. Related words failed to yield concreteness effects under any orienting condition. The results support the view that the presence or absence of concreteness effects in free recall depends on the relative salience of distinctive and relational information. This conclusion constrains theoretical explanations of the role of mental imagery in memory and cognition.
\end{abstract}

This paper concerns the role of mental imagery in the production of concreteness effects - that is, the relatively better memory for concrete than for abstract words. In contrast with the popularity of such studies during the 1960 s and 1970s, their frequency has waned in recent years. This decline is due in part to the advent of more sophisticated and exotic methods for examining mental imagery (see Paivio, 1971, 1986, for reviews). In addition, however, the shift away from such studies appears to have resulted from the tacit agreement that whatever else its role in cognition, imagery surely is the active ingredient in the production of concreteness effects in free recall of word lists (Marschark \& Cornoldi, 1990). Although several studies involving more complex materials have yielded results that raise problems for particular imagery theories (e.g., Brewer, 1975; Franks \& Bransford, 1972; Marschark \& Paivio, 1977), the reliability of the concreteness effect in list learning has not been questioned.

Portions of this research were conducted while $\mathbf{M}$. M. was supported by Grants NINCDS KO4-NS01267 and NIDCD K04-DC00028 and L. S. was supported by a grant from the Fulbright Foundation. We wish to thank Frank Bellezza, Carole Ernest, Douglas Nelson, and Ed Shoben for their extensive comments on earlier versions of this paper; Cesare Cornoldi, Johannes Engelkamp, Reed Hunt, and Hubert Zimmer for their thoughtful comments on the results; and Charles Huffman for his help in Experiment 2 and in revising this manuscript. Portions of this research were presented at the 1989 meeting of the Psychonomic Society in Atlanta, and the 1991 International Memory Conference in Lancaster, England. Correspondence can be addressed to M. Marschark, Department of Psychology, University of North Carolina at Greensboro, Greensboro, NC 27412, or via bitnet to marco@uncg.
Recently, however, several assumptions about the role of imagery in memory have been challenged by the finding that concreteness effects are not usually obtained in free recall of concrete and abstract texts (Marschark, 1985; Marschark, Warner, Thompson, \& Huffman, 1991; Wattenmaker \& Shoben, 1987; see Marschark et al., 1991, for consideration of apparent exceptions). Marschark (1985), for example, found that concrete sentences were recalled better than abstract sentences when both were presented as randomly organized lists, but that concreteness effects disappeared when the same sentences formed coherent paragraphs.

These findings are inconsistent with the assumption that concreteness effects result from the storage of images in long-term memory (Paivio, 1971, 1986; see Marschark, Richman, Yuille, \& Hunt, 1987, for discussion). According to the dual coding model, the relative ease of activating imaginal and verbal systems leads to a greater likelihood that concrete materials will be encoded in both imaginal and verbal forms, whereas abstract materials are likely to receive only verbal coding. At recall, the availability of two alternative memory codes is assumed to increase the relative probability of retrieving concrete material, thus producing the concreteness effect. However, it is difficult to see how such a model would explain concreteness effects' being obtained when sentences are randomly ordered but not when they are conceptually ordered, especially because coherent structure is assumed in the model to be relatively more important for concrete than for abstract verbal materials (Yuille \& Paivio, 1969).

Pursuing the apparent contradiction between studies involving text materials and earlier studies involving word 
lists, Marschark and Hunt (1989) reexamined the role of imagery in free and cued recall of concrete and abstract words. Their use of those relatively simple paradigms was motivated by the fact that dual coding explanations of the concreteness effect originally emerged from studies of that sort and by the celebrated robustness of the effect in such tasks. Marschark and Hunt, however, found that concreteness effects were obtained in cued recall only when orienting instructions induced relational processing at encoding and the encoded relational cues were presented at recall (cf. Tulving \& Thomson, 1973; see also Marschark \& Paivio, 1977). More importantly for the present purposes, they also found that concreteness effects were not obtained in free recall, even when an orienting task involved rating the imageability of to-be-remembered words (presented in pairs). This result was consistent with earlier findings of Morris and Stevens (1974) and Richardson (1975) and called into question any general explanation of concreteness effects that relies solely on the implicit generation of images during list learning.

The pattern of Marschark and Hunt's (1989) results across their five experiments suggests two other theoretical explanations of concreteness effects, both of which have long histories in the memory literature. One of these alternatives is that imagery provides a particularly effective means of interitem organization or integration. As a result, concrete words may be more easily or more strongly organized than abstract words, especially in tasks like paired-associate learning, and thus they are recalled better (Begg, 1972; Morris \& Stevens, 1974; cf. Surian \& Marschark, 1989).

The other alternative is that item-specific mental imagery may play a role in making concrete materials more distinctive in memory relative to abstract materials. Distinctiveness here refers to the relative contrast of any particular memory trace with others from which it must be discriminated in a particular recall context (Jacoby \& Craik, 1979). Distinctiveness, and hence memory, can be enhanced by more complete descriptions, more meaningful processing of relevant information, or the emphasis of distinguishing features. To the extent that concrete words are more likely than abstract words to receive such processing because of the availability of associated perceptual information in memory, they would be relatively more discriminable in memory and hence recalled better.

Both organizational and distinctiveness accounts of the role of imagery in memory have been largely rejected in their "pure" forms (Morris \& Stevens, 1974; see Paivio, 1971, 1986). Marschark and his colleagues (e.g., Hunt \& Marschark, 1987; Marschark et al., 1987; Marschark \& Surian, 1989a, 1989b), however, have suggested the possibility that the two mechanisms together might provide the best explanation of the presence and absence of concreteness effects in various memory tasks (see also Hunt \& Einstein, 1981; Morris \& Stevens, 1974). In this view, concreteness effects are assumed to reflect the enhanced distinctiveness or discriminability of items that have received enhanced, item-specific (e.g., imaginal) processing (cf. Stein \& Bransford, 1979). It is important, however, that the encoding of distinctive, item-specific information is assumed to have a significant impact on recall only when activated via encoded relational (contextual or associative) links (Humphreys, 1978). In paired-associate learning, for example, it is assumed that relational processing establishes links both between the pair members and (although perhaps to a lesser extent) between that pair and the encoding context (e.g., other pairs in the list; Hunt \& Marschark, 1987; cf. Begg, 1982). These links subsequently can be activated by appropriate cues at recall, at which point the relative distinctiveness of concrete words allows for better discrimination of those items than of abstract items within the search set.

It is essential to emphasize here that although both relational and item-specific processing are necessary to some extent in any memory task, either one may be more important for recall, depending on the nature of the situation. In memory for prose, for example, there is certainly distinctive, item-specific information associated with individual concrete and abstract sentences (as well as their component words), regardless of whether or not those sentences constitute coherent paragraphs. Comprehending and remembering connected text, however, places a premium on interitem, relational information, and itemspecific (word-, proposition-, or sentence-relevant) images are likely to be relatively less important. This situation contrasts with those of cued recall and free recall, which impose increasing importance on distinctive information in addition to relational information.

Given the relative roles of distinctive and relational information in memory, it is expected that concreteness effects in free recall will vary with the nature of the encoding context (Hunt \& Marschark, 1987). For example, intentional memory tasks, and especially laboratory tasks that involve some sort of organizational instructions, typically focus attention primarily on relational processing, as individuals explicitly attempt to interrelate to-beremembered items. This relational information, combined with the distinctiveness bestowed by item-specific imagery, would be expected to produce concreteness effects quite readily, and, in fact, such findings are the rule in intentional memory tasks (e.g., Rissenberg \& Glanzer, 1987; see Paivio, 1971, for a review).

Marschark and Surian (1989a), however, reported a study in which reliable concreteness effects were obtained on the first trial of an intentional memory task but disappeared on subsequent trials. This pattern of results, the exact opposite of the pattern to be reported below for incidental memory, did not result from any decrease in memory for concrete words, but from an increase in memory for abstract words. Apparently, under intentional memory instructions, subjects eventually were able to engage memory strategies for abstract words that balanced any a priori advantage held by concrete words.

Incidental memory paradigms such as those involving imageability or pleasantness ratings focus subjects' atten- 
tion primarily on item-specific information. If concreteness effects depend on the joint functioning of distinctive (imaginal) and relational information, their likelihood in incidental memory should be enhanced by conditions that facilitate relational processing (Hunt \& Einstein, 1981; Hunt \& Marschark, 1987). Consistent with this expectation, the pattern of results in the literature indicates that reliable concreteness effects in incidental recall are most likely to be obtained when there are multiple list presentations prior to recall or after multiple study-test trials (see Paivio, 1971). In contrast, reliable concreteness effects typically are not obtained in free-recall tasks that either limit subjects to item-specific orientations or disrupt relational processing (e.g., Marschark \& Hunt, 1989).

One apparent instance of the latter situation can be found in Paivio and Csapo's (1969) classic investigation of the effects of fast and slow presentation rates on memory for pictures, concrete words, and abstract words. In that study, subjects received eight study-test trials, a situation that normally would be expected to facilitate interitem relational processing. However, Paivio and Csapo presented their word lists in a different random order on each trial. This manipulation would be expected to result in a relative disruption of relational processing, although it clearly does not eliminate such processing under intentional learning instructions (see also Tulving, 1962). In fact, contrary to many citations of that report claiming otherwise, Paivio and Csapo did not obtain reliable concreteness effects in their free-recall conditions at any presentation rate.

The demonstration that concreteness effects in free recall depend on relational processing as well as itemspecific (imaginal) processing would impose important constraints on theories concerning the role of imagery in memory. In the following experiments, we therefore reexamined the robustness of concreteness effects in incidental free recall of word lists by using both indirect (Experiment 1) and direct (Experiment 2) manipulations of relational processing.

\section{EXPERIMENT 1}

In this experiment, we examined the hypothesis that the magnitude of the concreteness effects in incidental free recall is influenced by relational processing among to-beremembered words. Hunt and Marschark (1987) had suggested that the list context itself provides one source of relational information during learning in such a task, but they noted that a single presentation under incidental memory conditions would provide relatively little motivation or opportunity for subjects to engage in interitem relational processing. Providing an additional presentation of a list with the words in the same order, however, should increase the extent of relational processing among items in the list and between items and the whole list context.

The straightforward prediction tested in this experiment is that two list exposures prior to recall should increase the magnitude of the concreteness effect relative to a single list exposure. However, the manipulation of list exposures would confound the number of presentations with total viewing time. To reveal any effects of that confounding, stimulus lists were shown either once or twice at a rate of either 5 or $10 \mathrm{sec} /$ word. At the same time, we also wanted to ensure that subjects made use of visual imagery during study, and we therefore used the item-specific orienting task of having subjects rate the imageability of the words on both trials. The use of the imageability rating task leads the dual coding position to predict that concreteness effects should be obtained under all of the present conditions.

\section{Method}

Materials, Design, and Procedure. The stimulus list, presented via an overhead projector, was composed of 10 concrete and 10 abstract nouns (see the Appendix). These were randomly intermixed and preceded and followed by sets of 4 buffer items, with each set containing 2 concrete and 2 abstract nouns. All but 2 of the 10 abstract test nouns (rumor, routine) were listed in the Paivio, Yuille, and Madigan (1968) norms; their mean imagery rating was 3.52 $(S D=.82$ ). All but 2 of the concrete nouns (balloon, lamp) appeared in those norms; their mean imagery rating was $6.60(S D=$ .26). ${ }^{1}$ The complete design was a 2 ( 1 or 2 presentations) $\times 2(5$ or $10 \mathrm{sec} /$ item) $\times 2$ (concrete or abstract) mixed design, with concreteness as a within-subject variable.

The subjects rated the imageability of each word as it was presented. They were " informed" that normative ratings were needed to clarify findings from a previous study and were given standard imagery rating instructions (Paivio et al., 1968). Subjects who received a second presentation were told to try to form images again and check their first trial ratings. After all 28 words had been presented, the subjects were given blank sheets of paper and were asked to recall as many of the words as possible. They were given $5 \mathrm{~min}$ to complete the recall task.

Subjects. A total of 69 students participated in the study. All were enrolled in introductory psychology classes at the University of North Carolina at Greensboro and chose to participate as one option in a course research requirement. Eighteen students served in the $1 \mathrm{trial} / 10 \mathrm{sec}$ condition, and 17 served in each of the other (presentation rate $\times$ number of presentations) cells of the design. They were assigned to test conditions according to the session at which they chose to participate.

\section{Results and Discussion}

The results are depicted in Figure 1. The data were analyzed with a 2 ( 1 or 2 presentations) $\times 2$ ( 5 or $10 \mathrm{sec} /$ item) $\times 2$ (concrete or abstract) analysis of variance; unless otherwise noted, all results reported in this and the subsequent experiment were significant at or beyond the .05 level. The obvious pattern of results in Figure 1 was reflected in the findings that the slower presentation rate led to somewhat better memory (.36) than did the faster rate $(.31)\left[F(1,65)=3.87, M S_{e}=.03\right]$, and that, overall, concrete words $(.37)$ were remembered better than were abstract words $(.30)\left[F(1,65)=15.44, M S_{\mathrm{e}}=.01\right]$. The main effect of number of presentations was not significant $[F(1,65)=1.84]$, but the interaction of concreteness $\times$ presentations was reliable $[F(1,65)=11.16]$. The three-way interaction was not reliable $[F(1,65)<1]$, and, accordingly, post hoc Scheffé tests indicated that reli- 


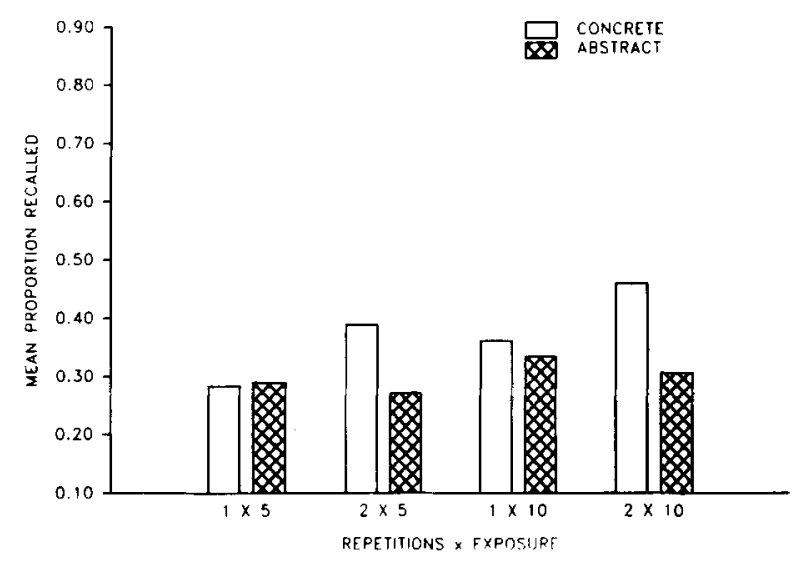

Figure 1. Mean proportions of concrete and abstract words recalled in Experiment 1.

able concreteness effects were obtained after two presentations at both the $5-\sec$ rate $[F(1,65)=9.84]$ and the 10 -sec rate $[F(1,65)=16.64]$, but not after a single presentation at either rate [both $F \mathrm{~s}(1,65)<1]$ (cf. Schwanenflugel, Akin, \& Luh, 1992). The power associated with the test for concreteness effects in the analysis of variance was $.99(\phi=.81$; Kirk, 1968).

In short, the results indicated that providing a second exposure to a list of concrete and abstract words increased the magnitude of the concreteness effect, whereas increasing total study time did not. A pilot study (reported by Marschark \& Surian, 1989a, 1989b) with different materials yielded the same result and also indicated that the addition of third and fourth exposures did not further increase the magnitude of the effect. In the present experiment, reliable concreteness effects were not obtained after a single trial despite the fact that the orienting task engaged subjects in rating the imageability of the words. Subjects performing such a task only once (in an incidental memory paradigm) would be unlikely to attend to interitem relationships, a situation working against the expression of concreteness effects (Humphreys, 1978; Hunt \& Marschark, 1987).

The present results replicate those of Morris and Stevens (1974) and Marschark and Hunt (1989), indicating that the facilitation of memory by mental imagery depends on its joint functioning with relational information. In the context of those and other recent studies (see Marschark \& Cornoldi, 1990), the results suggest that neither imagery nor any distinctiveness conferred by imagery is sufficient to account for the production of concreteness effects in free recall. Imagery does appear to be an important means of activating distinctive information in memory. The free-recall results of Experiment 1, however, cannot easily be explained in terms of a model that ascribes concreteness effects to the automatic or strategic formation of additional memory codes for concrete as opposed to abstract words (see, e.g., Paivio, 1986; Schwanenflugel et al., 1992).
The pattern of results obtained in this experiment also is consistent with findings recently reported by Nelson and Schreiber (1992). They evaluated the contributions of concreteness and associative structure to free recall, demonstrating over three experiments that concreteness and set size (i.e., the numeric diversity of single associates to a word) are independent. In their Experiment 3, Nelson and Schreiber also tested the proposal that the beneficial effects of concreteness in free recall depend on relational processing. As in the present experiment, they obtained reliably better memory for concrete words than for abstract words after two list presentations (.70 vs. .58) but not after a single one (.42 vs. .39).

\section{EXPERIMENT 2}

The results of Experiment 1 and those of Nelson and Schreiber (1992) provide support for the relationaldistinctive position. Both studies, however, involved indirect manipulations of relational context. The effects of explicit manipulations of relational processing on concreteness effects have not yet been addressed. Similarly, although the imagery rating task in Experiment 1 was intended to ensure subjects' use of imagery during study, the explicit effects of item-specific imagery under conditions more or less likely to promote relational processing were not addressed. Experiment 2 therefore was designed to contrast the effects of relational and imaginal processing in situations that varied in whether additional item-specific or relational information would be useful in free recall.

Experiment 2 involved a paradigm used by Hunt and Einstein (1981) in their demonstration that relational and item-specific processing together led to better free recall than either orientation alone. Using a categorization (relational) task and a pleasantness rating (item-specific) task, Hunt and Einstein found that item-specific processing of a list of related words and relational processing of a list of unrelated words led to better memory than did itemspecific processing of unrelated words, relational processing of related words, or doing either task twice on the same list.

The present experiment replicated the Hunt and Einstein (1981) paradigm in all essential respects, except that it involved the use of imagery ratings as the item-specific orienting task and included abstract as well as concrete words in both related and unrelated lists. To the extent that concreteness effects are affected by the crossing of relational and item-specific information, we expected that combinations of the relational task with the unrelated list and imagery ratings with either the relational task or the related list would lead to larger concreteness effects than would other combinations.

To our knowledge, no one has used categorized lists in imagery research before, and it was unclear whether imagery would remain an active ingredient of memory in the context of a well-structured set of words-that is, when relational organization is particularly salient. Generalizing from findings indicating that item-specific imagery 
does not play an essential role in memory for structured concrete texts (Marschark, 1985; Marschark et al., 1991), however, we expected that, overall, the effects of imagery would be attenuated with related words relative to unrelated words.

\section{Method}

Materials, Design, and Procedure. The related list was constructed using the Battig and Montague (1969) norms to equate category typicality as closely as possible. The concrete categories were fruits, insects, and musical instruments; the abstract categories were units of time, sciences, and elements. As in the Hunt and Einstein (1981) study, the unrelated list was constructed so that it constituted a parallel set of ad hoc categories, the structure of which would not be immediately obvious to subjects but would allow them to complete a categorization task without error. The ad hoc concrete categories were red things, wooden things, and metal things; the ad hoc abstract categories were scientific concepts, religious concepts, and political/governmental concepts. There were five exemplars in each category, so that each subject received a list of 30 test words making up 6 categories ( 3 concrete and 3 abstract). ${ }^{2}$ Each word was printed in the center of an index card, where it was preceded by a number from 1 to 30 . The decks were arranged consecutively for each subject for reasons that will be made clear below. The entire stimulus pool is presented in the Appendix.

The subjects were tested in small groups. Each group was tested on one of two random orders of either the related or the unrelated list. There were 10 test conditions, conducted in separate sessions, obtained by crossing the 2 list types with 5 orienting tasks: (1) rating imagery once, (2) categorizing once, (3) rating imagery twice, (4) categorizing twice, and (5) rating imagery once and categorizing once. The order of the 2 tasks in the last condition was counterbalanced across testing sessions.

The imagery rating instructions were identical to those used in Experiment 1 . The 7 -point rating scale remained in view throughout the orienting task, and the subjects wrote word numbers and their ratings on a blank sheet of paper. Subjects who did the rating task twice were told to repeat their evaluations independently of the first, so that we could examine their consistency across task repetitions. Their first set of ratings was not available while they were performing the second rating task.

The categorization instructions were similar to those used by Hunt and Einstein (1981). The subjects first sorted the cards according to the category headings listed above; the appropriate list of six categories remained in view throughout the orienting task. When they had completed their sortings, the subjects wrote the category names on a blank sheet of paper and then wrote the numbers of the words (not the words themselves) under the category names, corresponding to the way in which they had been sorted. The use of numbers here was intended to avoid having subjects write the words and thus receive extra exposure to them relative to the imagery rating task. Subjects who did the categorization task a second time were told to shuffle their cards and repeat their sortings independently of the first trial, so that we could examine their consistency across task repetitions; their first listings were not available during the second categorization task. The sorting data were not evaluated, although casual examination of them indicated perfect category correspondence with both related and unrelated (ad hoc) lists.

The complete design was thus a 5 (orienting condition) $\times 2$ (related or unrelated list) $\times 2$ (concrete or abstract) mixed factorial, in which only concreteness was a within-subject variable. Following the orienting task, the subjects were given an unexpected freerecall task. Five minutes were allotted for recall.

Subjects. There were 120 subjects in the complete design, 12 in each of the 10 list type $x$ orienting task conditions. They were randomly assigned to the conditions according the session at which they chose to participate. All were drawn from the same pool as in the previous experiment.

\section{Results and Discussion}

As might be expected, it is rather difficult to construct abstract categories for tasks such as this, and we had some concern about the generalizability of the materials actually employed. Two sets of analyses were therefore performed on the recall data, one with subjects as the random factor and another with words as the random factor.

Overall, the related list was recalled better than the unrelated list. If one examines recall of the former, it can be seen in Figure 2 that there were no significant concreteness effects in any condition (although the power associated with the test was $.99, \phi=4.99$ ). In contrast, recall of the unrelated list produced large and reliable concreteness effects in the categorization once $[F(1,110)=19.29]$ and categorization twice $[F(1,110)=$ $22.80]$ conditions, according to post hoc Scheffé tests. ${ }^{3}$ The fact that the magnitude of the concreteness effect did

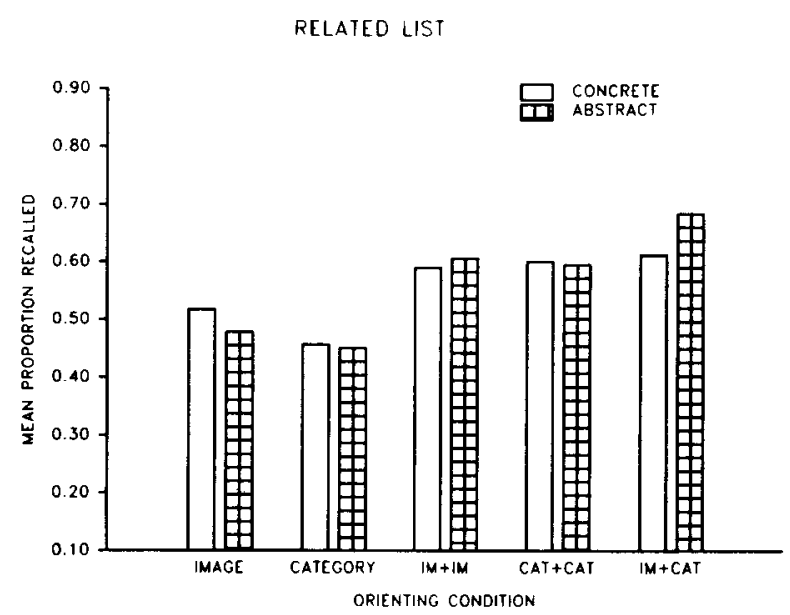

UNRELATED LIST

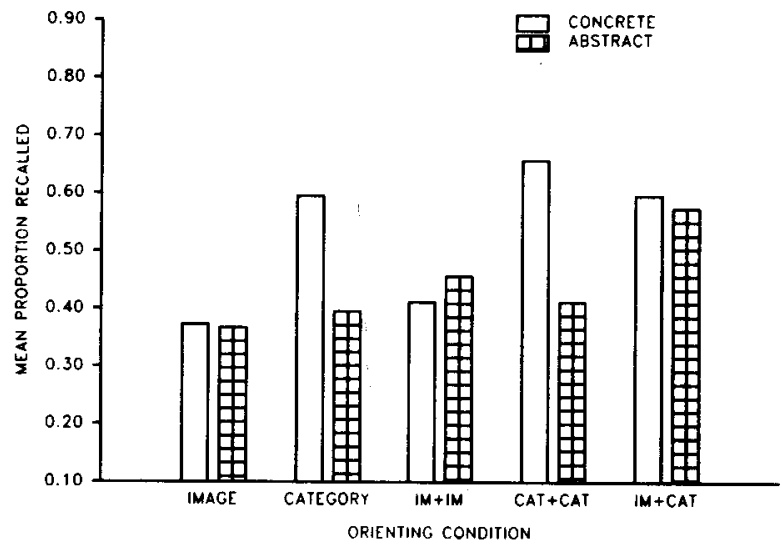

Figure 2. Mean proportions of concrete and abstract words recalled from related and unrelated lists in Experiment 2. 
not increase from one to two categorizations suggests that although the production of concreteness effects requires some minimum amount of relational processing, as relational information becomes more salient it can contribute to the recall of abstract as well as concrete materials (Franks \& Bransford, 1972; Marschark, 1985; Marschark \& Surian, 1989a, 1989b).

The pattern of results above yielded main effects for both subjects and words (respectively) of list type $\left[F(1,110)=12.30, M S_{\mathrm{c}}=.03 ;[F(1,140)=24.88\right.$, $\left.M S_{\mathrm{e}}=.62\right]$ and orienting condition $[F(4,110)=8.97$; $F(4,140)=7.61]$. The overall effect of concreteness proved reliable in the subject analysis $[F(1,110)=7.28$, $\left.M S_{\mathrm{e}}=.01\right]$, but not in the item analysis $[F(1,140)=$ 2.61 ]. In both subject and item analyses, there were interactions of condition $\times$ list type $[F(4,110)=2.64$; $F(4,140)=7.95]$ and concreteness $\times$ list type $[F(1,110)$ $=10.49 ; F(1,140)=3.24, p<.08]$ (see Figure 2). Only in the subject analyses were the interactions of condition $X$ concreteness $[F(4,110)=4.98]$ and condition $\times$ concreteness $\times$ list type $[F(4,110)=3.74]$ reliable.

Because of the importance of the finding of concreteness effects in the two conditions involving categorization of the unrelated list, replications of those conditions were conducted. There were 11 subjects in one group (discarding 1 who wrote the exemplars) and 12 in the other. Once again, both conditions yielded reliable concreteness effects $\left[F(1,21)=22.60, M S_{e}=.01\right]$. Recall was higher after two categorizations than after one $[F(1,21)=15.60$, $\left.M S_{\mathrm{e}}=.02\right]$, but the interaction with concreteness was not reliable $[F(1,21)=2.44]$.

The failure to obtain concreteness effects in any of the four independent imagery conditions of this experiment is consistent with the findings of Experiment 1 and those of Marschark and Hunt (1989, Experiment 3). Interestingly, requiring subjects in the rating-twice condition to repeat their imageability ratings independently appeared to maintain their focus on item-specific information to a greater extent than did simply asking them to check over their initial ratings (as in Experiment 1). Subjects who rated the unrelated list twice in Experiment 2 did not produce reliable concreteness effects here in either the original two conditions of Experiment 2 or the replication, whereas those in Experiment 1 produced a reliable effect on the second trial and those in the pilot study for that experiment produced reliable effects after two, three, and four exposures (with nonspecific orienting instructions).

The present results clearly create a basic difficulty for any model that ascribes concreteness effects to the likelihood of subjects' generating mental images during list learning. At the same time, however, the failure to obtain any appreciable concreteness effects after rating the imageability of a related list is rather surprising from the perspective of the relational-distinctive position.

In general, the lack of concreteness effects in all of those conditions appears to have resulted from the improved recall of abstract words, a finding consistent with those of Marschark (1985) and Marschark and Surian (1989a). Some insight into these results can be obtained through examination of the extent to which subjects clustered their recall by category in their protocols. Because the calculation of ARC scores with a mixed design is problematic, we simply counted the number of words recalled consecutively from each category. The clustering analyses thus are not corrected for chance pairing of same-category items and may overestimate clustering in favor of conditions in which recall was higher.

The mean frequencies of category repetitions in recall are depicted in Figure 3, where it can be seen that clustering was more likely in the related than in the unrelated list $\left[F(1,109)=49.54, M S_{\mathrm{e}}=4.49\right]$ and varied across the orienting tasks $[F(4,109)=6.54]$. As is evident in the figure, abstract words were more likely to be recalled in categories than were concrete words in the related list but not the unrelated list, a pattern that produced a reliable list type $\times$ concreteness interaction $[F(1,109)=$ $\left.14.47, M S_{e}=2.01\right]$. Although there was no overall effect of concreteness in clustering $[F(1,109)<1]$, relative differences in clustering of concrete and abstract words across the orienting conditions produced a reliable con-

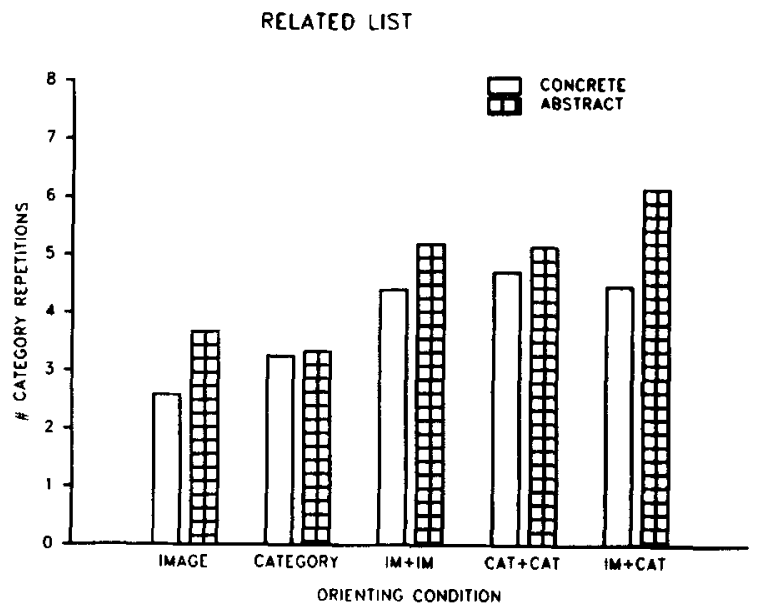

UNRELATED LIST

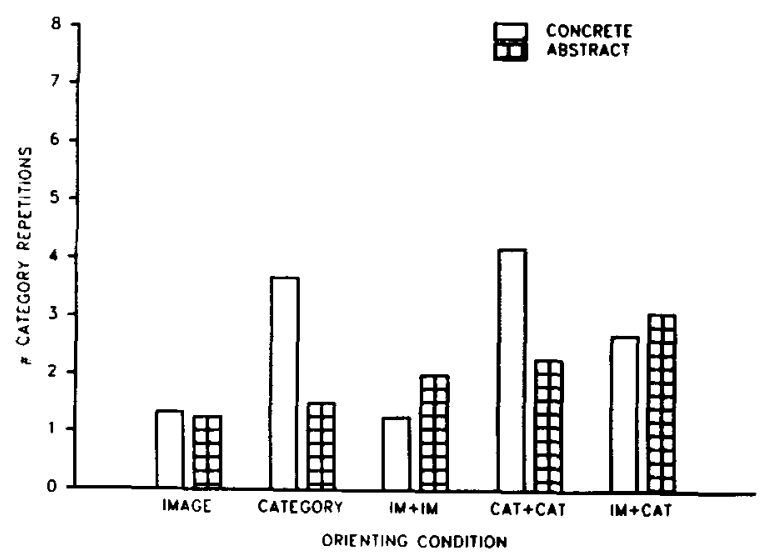

Figure 3. Mean frequencies of category repetition in recall from related and unrelated lists in Experiment 2. 
dition $\times$ concreteness interaction $[F(4,109)=5.07]$. Concrete words were clustered in recall more often than abstract words only in the two conditions in which unrelated words had been categorized-the two conditions that produced concreteness effects.

In summary, the results of this experiment suggest that the contribution of imagery to the free recall of unrelated word lists is largely one of increasing the distinctiveness of the items in memory. In concert with explicit relational processing at encoding and the utilization of relational in formation at retrieval, this enhanced distinctiveness leads to reliable concreteness effects (Marschark \& Hunt, 1989). At the same time, however, there are clearly nonimaginal, relational processes available to subjects that allow them to organize abstract materials in a way that balances the effects of item-specific imagery. Thus, concreteness effects are attenuated or eliminated while recall of abstract materials increases to the level of concrete materials (see also Marschark, 1985; Marschark \& Paivio, 1977). Like the results of Experiment 1, the results of this experiment indicate that such processes may not be apparent after a single presentation of an unrelated list but quickly become so when additional opportunities for relational processing are available.

\section{GENERAL DISCUSSION}

The present experiments were prompted by Marschark and Hunt's (1989) finding that concreteness effects were not obtained in the free recall of words acquired in a paired-associate learning task, even when subjects were engaged in rating the imageability of the words. In contrast, concreteness effects were obtained in free recall when subjects performed a relational processing task. Marschark and Hunt took those results as support for the hypothesis that concreteness effects in list learning depend on the joint processing of relational and distinctive information (Humphreys, 1978; Hunt \& Einstein, 1981). From that perspective, their failure to obtain concreteness effects in free recall could have resulted from the use of only a single learning trial in combination with orienting tasks that did not encourage relational processing either between pair members or between the individual pairs and the list context. Marschark and Hunt speculated, however, that the likelihood of concreteness effects in free recall could increase with additional learning trials.

The results of the present study support that prediction, replicating and extending the previous findings with more standard free-recall tasks. In Experiment 1, reliable concreteness effects were obtained after two presentations at both 5- and 10-sec/item presentation rates but not after a single presentation at either rate (see also the pilot study reported by Marschark \& Surian, 1989a, 1989b). The fact that this reliable interaction was obtained in the context of an imageability rating task means that this result raises problems for any theory that explains concreteness effects in terms of the likelihood of generating images during encoding.
In a paradigm designed to demonstrate the independent contributions to recall of item-specific and relational information, Experiment 2 indicated that repeated presentations of an unrelated list only contribute to the concreteness effect when subjects engage in relational processing. Such processing might ensue, and concreteness effects are in fact obtained, when a list is repeatedly presented or when subjects are engaged in relational tasks, but neither apparently occurs when subjects are engaged in itemspecific tasks (Experiment 2; Huffman, 1991; Marschark \& Hunt, 1989, Experiment 3) or when interitem relational information is disrupted (Marschark \& Hunt, 1989, Experiment 5; Paivio \& Csapo, 1969).

Categorized lists apparently have not been used previously in studies of imagery and concreteness effects in memory. At least in the case of our laboratory, this is because coherent categories of abstract words are difficult to construct (a potentially interesting phenomenon in its own right). In Experiment 2, related concrete and abstract words did not yield concreteness effects, regardless of whether they were presented once or twice and regardless of whether subjects were engaged in an itemspecific or a relational task (cf. Hunt \& Einstein, 1981). As was evident in Figures 2 and 3, these findings apparently derived from subjects' making use of category information in their recall of abstract words even more frequently than they did with concrete words, independently of the orienting task. At the same time, the magnitude of the concreteness effect did not increase with a second categorization in Experiment 2. This finding suggests that although some amount of relational processing is necessary for the production of a concreteness effect, sufficient attention to such information (not surprisingly) can facilitate memory of abstract as well as concrete materials (Marschark, 1985; Marschark et al., 1991; cf. Paivio, 1991).

Considered together with the findings of Marschark and Hunt (1989), the present results suggest that concreteness effects in free recall cannot be explained fully in terms of the relative availability of dual and single memory codes for concrete and abstract words, respectively (e.g., Paivio, 1991). Instead, the effect appears to depend on the processing of both item-specific and relational information at the time of encoding and the reactivation of both at retrieval (Begg, 1972, 1982). Imagery, from this point of view, provides a particularly powerful mechanism for enhancing the distinctiveness of verbal materials and hence is typically correlated with recall (Berrian, Metzler, Kroll, \& Clark-Myers, 1979; Paivio, 1971; Rubin, 1980). Imagery, however, is clearly not a sufficient explanation for concreteness effects in memory.

This general conclusion goes beyond the recall of word lists, for it is also consistent with the failure to obtain concreteness effects in the comprehension (Schwanenflugel \& Shoben, 1983) or recall of concrete and abstract texts (Marschark, 1985; Wattenmaker \& Shoben, 1987), both situations in which relational information is preeminent. In fact, the finding that salient relational organization can 
particularly benefit abstract materials is consistent with the frequent, if small, advantage observed in recall of abstract over concrete paragraphs (Marschark, 1985; Marschark et al., 1991; see also Paivio \& Walsh, as cited in Paivio, 1991). Imagery also might provide an extraordinary mechanism for relational processing, through interactive imagery, although findings obtained by Surian and Marschark (1989) do not appear to support this possibility.

Paivio (1991, p. 26) suggested that one argument against the relational-distinctive framework is that relational processing is invoked to explain both the presence and the absence of concreteness effects in various tasks. In particular, Marschark (1985) argued that the greater importance of relational information relative to itemspecific imagery in text memory can eliminate the concreteness effect, whereas Marschark and Hunt (1989) claimed that the expression of concreteness effects depends on relational processes at both study and retrieval in cuedand free-recall tasks. Far from representing an "inconsistency" in the relational-distinctive framework, we see these explanations as two sides of the same coin, both indicating the importance of relational processing in memory but also emphasizing its relative role in various recall tasks.

One alternative account of the presence and absence of concreteness effects in various tasks is the context availability model. Schwanenflugel and Shoben (1983) suggested that the number of possible contexts for a word might be the locus of several findings previously ascribed to imagery, including concreteness effects in memory. More recently, Schwanenflugel and her colleagues (Schwanenflugel, Harnishfeger, \& Stowe, 1988; Schwanenflugel et al., 1992) have demonstrated that context availability ratings do not predict either comprehension or lexical decisions, and they argue, instead, "that abstract words are comprehended more poorly because persons take longer to retrieve readily available information associated with them (assessed via ratings of context availability and not via word associations)" (Schwanenflugel, personal communication, December 17, 1991; cf. Nelson \& Schreiber, 1992).

Schwanenflugel et al. (1992) recently tested the context availability hypothesis in a study of free recall of concrete and abstract words. They varied the rated concreteness of their stimuli while controlling context availability (as indexed by normative ratings) and obtained a reliable concreteness effect in incidental recall following an imagery rating task but not following a context availability rating task. Those results were interpreted as indicating that imagery is used strategically in recall when "prior contextual knowledge" appears insufficient (see also Helstrup, 1987).

With this added assumption, the context availability model retains context effects as the explanation of concreteness effects in comprehension and memory for sentences and paragraphs but invokes imagery as the explanation for concreteness effects in memory for word lists.
However, the model does not provide any explanation beyond dual memory codes of why imagery improves memory when it is strategically employed, and it is clear from the results above that dual memory codes are insufficient to explain concreteness effects in free recall. ${ }^{4}$

In our view, the present results can be explained best in terms of the joint functioning of relational and distinctive information in memory. The relational-distinctive model generally appears to account for a broader range of imagery-related results than does either the dual coding or the context availability model (see Marschark \& Cornoldi, 1990; Marschark et al., 1987, for reviews). None of the three models, however, has been uniformly successful in its a priori predictions. At this point, we believe that there are relatively few theoretical stumbling blocks that prevent the three models from providing a common account of imagery and concreteness within the relationaldistinctive rubric. Hopefully, such a unified account will prove more powerful than any one of the models alone.

\section{REFERENCES}

Battig, W. F., \& Montague, W. E. (1969). Category norms for verbal items in 56 categories: A replication and extension of the Connecticut category norms. Joumal of Experimental Psychology Monographs, 80 (3, Pt. 2).

BEGG, I. (1972). Recall of meaningful phrases. Journal of Verbal Leaming \& Verbal Behavior, 11, 431-439.

BEGG, I. (1982). Imagery, organization, and discriminative processes. Canadian Journal of Psychology, 36, 273-290.

Berrian, R. W. Metzler, D. P., Kroll, N. E. * Clark-Meyers, G. M. (1979). Estimates of imagery, ease of definition, and animateness for 328 adjectives. Journal of Experimental Psychology: Human Learning \& Memory, 5, 435-447.

BREWER, W. F. (1975). Memory for ideas: Synonym substitution. Memory \& Cognition, 3, 458-464.

Franks, J., Bransford, J. (1972). The acquisition of abstract ideas. Joumal of Verbal Leaming \& Verbal Behavior, 11, 311-315.

HelsTRUP, T. (1987). One, two, or three memories? A problem-solving approach to memory for performed acts. Acta Psychologica, 66, 37-68.

HUFFMAN, C. (1991). The contributions of ease of imagery and image vividness to concreteness effects in memory. Unpublished master's thesis, University of North Carolina, Greensboro.

HuMPHREYs, M. S. (1978). Item and relational information: A case for context independent retrieval. Journal of Verbal Learning \& Verbal Behavior, 17, 175-188.

Hunt, R. R., Einstein, G. O. (1981). Relational and item-specific information in memory. Journal of Verbal Learning \& Verbal Behavior, 15, 559-566.

Hunt, R. R., \& MARSChaRK, M. (1987). Yet another picture of imagery: The role of shared and distinctive information. In $\mathbf{M}$. Pressley \& M. McDaniel (Eds.), Imagery and related mnemonic processes (pp. 129-150). New York: Springer-Verlag.

JACOBY, L. L., \& CrAIK, F. I. M. (1979). Effects of elaboration of processing at encoding and retrieval: Trace distinctiveness and recovery of initial context. In L. S. Cermack \& F. I. M. Craik (Eds.), Levels of processing in human memory (pp. 1-21). Hillsdale, NJ: Erlbaum.

KIRK, R. E. (1968). Experimental design: Procedures for the behavioral sciences. Belmont, CA: Brooks/Cole.

MARSCHARK, M. (1985). Imagery and organization in the recall of prose. Journal of Memory \& Language, 24, 734-745.

Marschark, M., CoRnOldi, C. (1990). Imagery and verbal memory. In C. Cornoldi \& M. McDaniel (Eds.), Imagery and cognition (pp. 133-182). New York: Springer-Verlag.

Marschark, M., HUNT, R. R. (1989). A reexamination of the role 
of imagery in learning and memory. Joumal of Experimental Psychology: Learning, Memory, \& Cognition, 15, 710-720.

Marschark, M., \& Paivio, A. (1977). Integrative processing of concrete and abstract sentences. Journal of Verbal Learning \& Verbal Behavior, 16, 217-231.

Marschark, M., Richman, C. L., Yuille, J. C., Hunt, R. R. (1987). The role of imagery in memory: On shared and distinctive information. Psychological Bulletin, 102, 28-41.

MARSChARK, M., SURIAN, L. (1989a, November). Why are there (sometimes) concreteness effects in free recall? Paper presented at the meeting of the Psychonomic Society, Atlanta, GA.

MARSCHARK, M., \&URIAN, L. (1989b). Why does imagery improve memory? European Journal of Cognitive Psychology, 1, 251-263.

Marschark, M., Warner, J., Thompson, R., Hufman, C. (1991). Concreteness, imagery, and memory for prose. In R. Logie \& M. Denis (Eds.), Imagery in human cognition (pp. 194-207). Amsterdam: Elsevier.

MoRRIs, P. E., \& STEVENS, R. (1974). Linking images and free recall. Journal of Verbal Learning \& Verbal Behavior, 13, 310-315.

Nelson, D. L., SCHREIBER, T. A. (1992). Word concreteness and word structure as independent determinants of recall. Journal of Memory \& Language, 31, 237-260.

PaIvio, A. (1971). Imagery and verbal processes. New York: Holt, Rinehart \& Winston.

PAIvio, A. (1986). Mental representations: A dual coding approach. Oxford: Oxford University Press.

Paivio, A. (1991). Dual coding theory: Retrospect and current status. Canadian Journal of Psychology, 45, 255-287.

Paivio, A., Csapo, K. (1969). Concrete-image and verbal memory codes. Joumal of Experimental Psychology, 80, 279-285.

Paivio, A., Yuille, J. C., \& Madigan, S. (1968). Concreteness, imagery, and meaningfulness values for 925 nouns. Journal of Experimental Psychology Monographs, 76 (1, Pt. 2), 1-25.

RichARDSON, J. T. E. (1975). Imagery and deep structure in the recall of English nominalizations. British Journal of Psychology, 66, 333-339.

Rissenberg, M., \& Glanzer, M. (1987). Free recall and word finding ability in normal aging and senile dementia of the Alzheimer's type: The effect of item concreteness. Journal of Gerontology, 42, 318-322.

RuBIN, D. C. (1980). 51 properties of 125 words: A unit analysis of verbal behavior. Journal of Verbal Learning \& Verbal Behavior, 19. 736-755.

SChwanenflugel, P., Akin, C., \& Luh, W.-M. (1992). Context availability and the recall of abstract and concrete words. Memory \& Cognition, 20, 96-104.

SChwanenflugel, P. J., Harnishfeger, K. K., \& Stowe, R. W. (1988). Context availability and lexical decisions for abstract and concrete words. Journal of Memory \& Language, 27, 499-520.

Schwanenflugel, P., Shoben, E. J. (1983). Differential context effects in the comprehension of concrete and abstract prose. Journal of Experimental Psychology: Learning, Memory, \& Cognition, 9, 82-102.

Stein, B., \& Bransford, J. D. (1979). Constraints on effective elaboration: Effects of precision and subject generation. Journal of Verbal Learning \& Verbal Behavior, 18, 769-778.

SURIAN, L., MARSCHARK, M. (1989). Imagery and memory: Effects of semantic relations and concreteness (Research Bulletin No. 32). University of North Carolina, Greensboro, Language Research Group.

TuLvING, E. (1962). Subjective organization in free recall of "unrelated" words. Psychological Review, 69, 344-354.

Tulving, E., ThOMson, D. (1973). Encoding specificity and retention processes in episodic memory. Psychological Review, 80, 352-373.

WATtenmaker, W. D., SHObEN, E. J. (1987). Context and the recallability of concrete and abstract sentences. Joumal of Experimental Psychology: Learning, Memory, \& Cognition, 13, 140-150.

Yuille, J. C., \&AIvio, A. (1969). Abstractness and the recall of connected discourse. Journal of Experimental Psychology, 82, 467-471.

\section{NOTES}

1. The stimuli for this experiment were selected from among those we were using in other studies at the time. Why they were not all selected from the Paivio et al. (1986) norms remains a mystery.

2. The subjects were supposed to receive six exemplars from each category ( 36 words). Owing to a collation error, however, half of the subjects only received five exemplars in one category. We therefore randomly deleted one exemplar from each of the other categories, and we scored recall with those items deleted.

3. Although the concreteness effect was not reliable in the imagery plus categorization group, there was a trend in the data such that 4 of the 6 subjects in the imagery-then-categorization subgroup recalled more concrete than abstract words, and in 1 case recall was equal. Meanwhile, only 1 of the 6 subjects in the categorization-then-imagery subgroup recalled more concrete words than abstract words, and in 4 cases, recall was equal.

4. Discussions with Paula Schwanenflugel have failed to reveal why Schwanenflugel et al. (1992, Experiment 2) obtained a concreteness effect after an imagery rating task, whereas in the present experiments we did not. The only apparent difference in method resides in our use of visual presentation and their use of auditory presentation. Although it is unclear why mode of presentation would affect concreteness effects, several hypotheses are currently under investigation.

\section{APPENDIX}

Stimuli for Experiment 1

\begin{tabular}{ll}
\hline Concrete & Abstract \\
\hline newspaper & ignorance \\
ticket & kindness \\
lake & chance \\
lamp & attitude \\
chair & routine \\
snake & rumor \\
iron & mood \\
doctor & advantage \\
balloon & spirit \\
factory & interest \\
\hline
\end{tabular}

Stimuli for Experiment 2

Concrete

Related List

Fruits: peach, orange, banana, grape, strawberry Musical instruments: cymbal, horn, cornet, viola, cello Insects: caterpillar, centipede, ant, wasp, locust

Abstract

Time: century, month, decade, week, millisecond Elements: calcium, uranium, magnesium, zinc, helium Sciences: geology, physiology, botany, zoology, genetics

\section{Unrelated List}

Concrete

Red things: tomato, blood, rose, apple, cherry

Metal things: fork, submarine, car, pliers, stove

Wooden things: arrow, pencil, desk, floor, violin

Abstract

Religion: belief, devotion, miracle, soul, proverb Science: precision, astronomy, theory, botany, research Government: politics, legislation, election, democracy, candidacy 